\title{
Search for Transiting Exoplanets with HATNet
}

\author{
G. Á. Bakos ${ }^{1}$, R. W. Noyes ${ }^{1}$, G. Kovács ${ }^{2}$, D. W. Latham ${ }^{1}$, G. Torres ${ }^{1}$, \\ D. Sasselov ${ }^{1}$, A. Pál ${ }^{1,3}$, B. Sipőcz ${ }^{3,1}$ and Gábor Kovács ${ }^{1}$ \\ ${ }^{1}$ Harvard-Smithsonian Center for Astrophysics, \\ 60 Garden street, MA 02138, USA \\ email: gbakos@cfa.harvard.edu \\ ${ }^{2}$ Konkoly Observatory, Budapest \\ ${ }^{3}$ Loránd Eötvös University, Budapest
}

\begin{abstract}
HATNet is a network of six identical, fully automated wide field telescopes, four of which are located in Arizona, and two at Hawaii. The purpose of the network is to search for transiting extrasolar planets around relatively bright stars $(8<I<12)$. The longitudinal coverage of 3.5 hours greatly enhances transit detection efficiency. HATNet has been operational since 2004, and has taken more than $1 / 2$ million science frames at 5 -min integrations, covering about $7 \%$ of the sky. Photometric precision reaches $3 \mathrm{mmag}$ rms at $5.5 \mathrm{~min}$ cadence at $I \approx 8$, and is $1 \%$ at $I \approx 11.3$. Hundreds of transit candidates have been detected in the data, and have been subject to vigorous follow-up by various $1 \mathrm{~m}$-class facilities, both spectroscopy and follow-up photometry. A fraction of the candidates that have survived these steps as not being false alarms have been observed by high resolution and precision spectrographs (primarily Keck/HIRES), to confirm their planetary nature and characterize their properties. So far nine transiting planets have been reported, making HATNet a very successful survey.
\end{abstract}

\section{Brief History}

Design and construction of the HAT prototype instrument was initiated by Bohdan Paczyński in 1999. The original purpose was to monitor the Northern sky for all-sky variability, in a similar fashion to the ASAS project (Pojmański 1997) that was conducting a survey in the Southern hemisphere. After successful tests at Konkoly Observatory, the instrument was moved to Kitt Peak National Observatory, and was remotely operated in the period of 2001-2003 (Bakos et al. 2002). In the following year HAT-1 was de-comissioned, and the instrument underwent significant re-design. An array of new generation HAT instruments (called HAT-5, 6, 7) was installed at the Smithsonian Astrophysical Observatory's (SAO) Fred Lawrence Whipple Observatory (FLWO) in 2003/2004, thus establishing HATNet's first station. Two more instruments (called HAT-8, HAT-9) were installed on Mauna Kea, Hawaii, on SAO's Submillimeter Array (SMA) grounds in late 2004, and HATNet gained longitudinal coverage. Finally, in 2005 yet another HAT (called HAT-10) was installed to FLWO. We note that the Wise HAT (WHAT) instrument is very similar to the HATNet instruments, and was installed in 2003 at Wise Observatory in Israel. Another part of HATNet is TopHAT, a slightly bigger $(0.25 \mathrm{~m}, \mathrm{f} / 5)$ instrument at FLWO, with the goal of performing photometry follow-up observations on HATNet candidates. TopHAT has been operational since 2006 . 


\section{Instrument Parameters}

HATNet instrumentation has undergone significant changes over time. The HAT telescope optic is a $0.11 \mathrm{~m}$ diameter $\mathrm{f} / 1.8$ Canon lens. Until 2007 we used Cousins I-band filters and $2 \mathrm{~K} \times 2 \mathrm{~K}$ front-illuminated Apogee AP10 CCDs with $14^{\prime \prime}$ pixels, yielding a wide, $8^{\circ} \times 8^{\circ}$ field. Recently some of the CCDs have been replaced with Apogee U16m $4 \mathrm{~K} \times 4 \mathrm{~K}$ detectors and Sloan $r$ filters, yielding $9^{\prime \prime}$ per pixel resolution and a $10.6 \times 10.6^{\circ}$ field. The CCDs and lenses are attached to a horseshoe mount, protected by a clamshell dome, and all devices are under control of a single PC running Linux. The original instrument description is presented in Bakos et al. (2002).

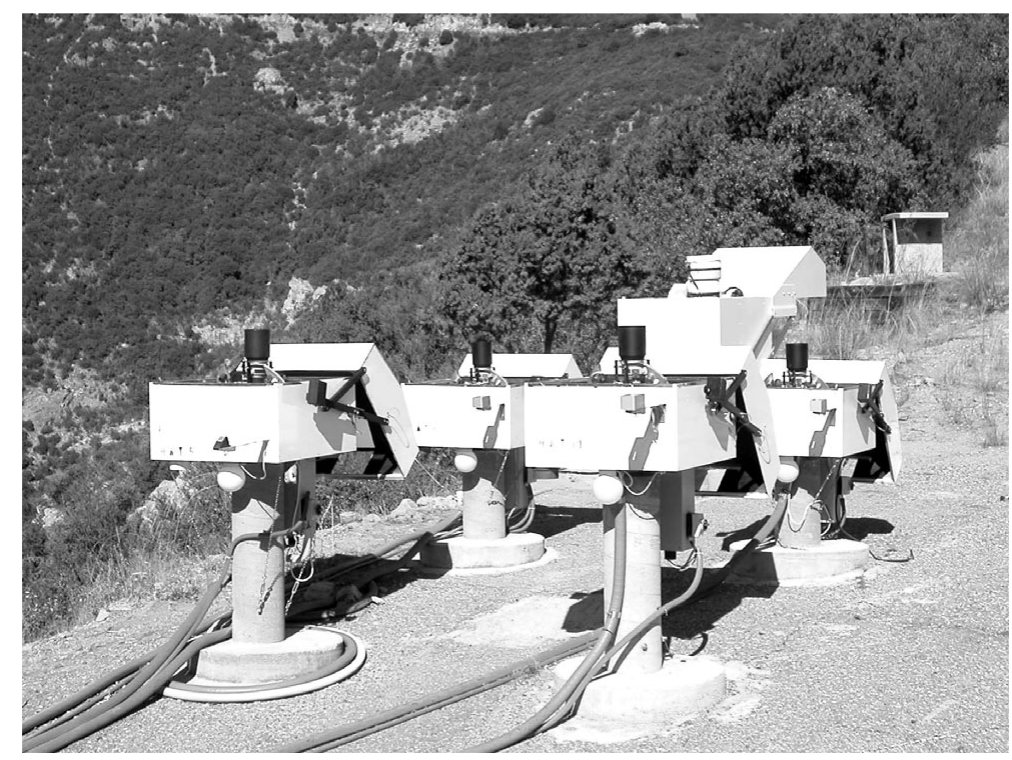

Figure 1. HATNet station at FLWO, Arizona, hosting HAT-5, 6, 7 and HAT-10, plus TopHAT (in the background). The clam-shell domes are normally closed in the daytime, and protect all the equipment inside: the Canon $200 \mathrm{~mm} \mathrm{f} / 1.8$ lenses, $4 \mathrm{~K} \times 4 \mathrm{~K}$ CCDs and horseshoe mounts.

\section{Operations}

Routine nightly operations start with cooling down the CCD, opening the dome, and taking skyflat calibration frames. After nautical twilight HAT proceeds to monitor a selected field by staring at it whenever the field is visible (taking into account horizon limits, moon distance, etc). If the primary field is not visible, HAT observes a secondary field. All valuable time from dusk to dawn is used for taking 5-minute exposures. More details on operations can be found in Bakos et al. (2004), including the psf-broadening technique, whereby the telescope follows a prescribed pattern during the exposures to smooth the stellar profiles, and decrease the effect of front-illuminated pixel structure on the photometry.

As of mid-2008, the suite of instruments has automatically opened on more than 1300 nights at FLWO, and more than 1100 nights for Mauna Kea (successful opening is defined when more than 40 frames are taken at 5 -min cadence). We have gathered more than $1 / 2$ million science frames on $\sim 60$ fields, corresponding to $7 \%$ sky coverage. The total data volume is $20+$ Terabytes, including secondary data-products (such as photometry). Data are hosted at the Harvard-Smithsonian Center for Astrophysics (CfA) on our custombuilt server computers. 


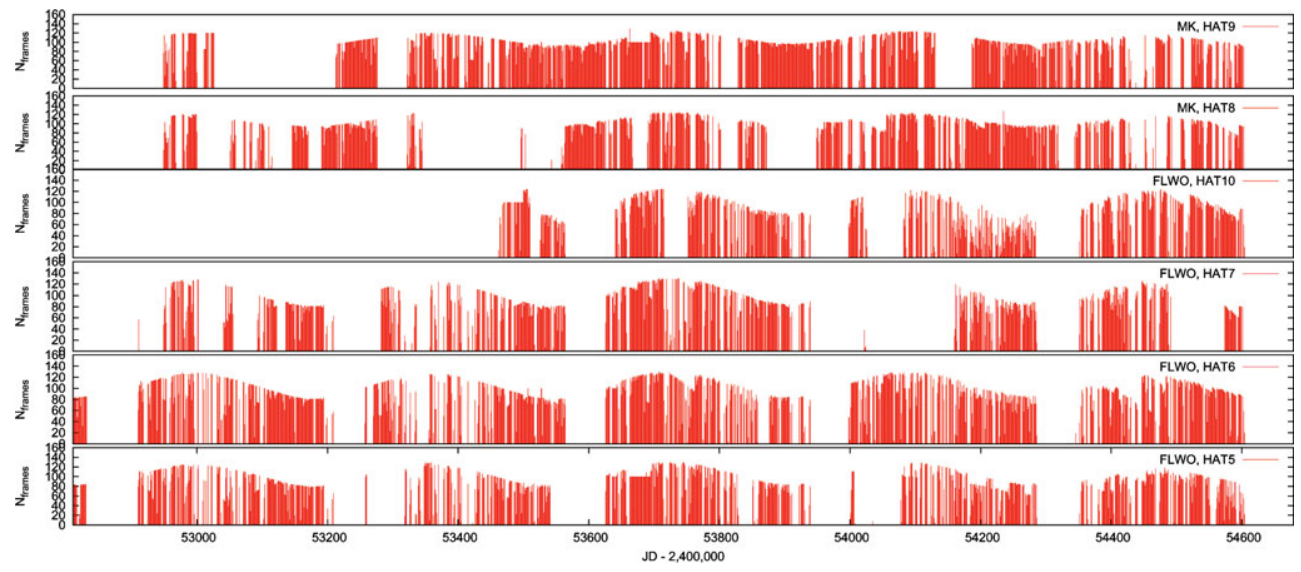

Figure 2. Observing statistics of the HATNet telescopes as a function of Julian Date. The vertical axis shows the number of 5-minute exposures taken per night. The dominant features are the annual variation of dark time, and the Arizona monsoon (e.g. for HAT-5, approx 1.5 month gaps).

\section{Data Analysis and Search for Transits}

Images are corrected for overscan, bias, dark and flatfield using IRAF procedures. Astrometry with respect to the 2MASS (Skrutskie et al. 2006) catalogue is performed as described in Pál \& Bakos (2006). Aperture photometry is carried out in 3 apertures on the fixed centroid positions of stars based on the 2MASS catalogue and the astrometric transformation. Raw magnitudes are transformed to the (arbitrary) magnitude scale of a selected reference frame. The resulting light curves are searched for shallow and periodic transit signals using the Box Least Squares (BLS) fitting method (Kovács, Zucker, \& Mazeh 2002). However, this search is hindered by the existence of systematic variations (red noise) in our light curves (Pont, Zucker, \& Queloz 2006). Thus, we developed the Trend Filtering Algorithm (TFA), which largely corrects trends by using 1000 "template" stars within the same field (Kovács, Bakos, \& Noyes 2005). To further beat down the red noise, we have developed an External Parameter Decorrelation technique (briefly described in Bakos et al. 2007b) which attempts to take out deviations from the median of the light curves, by correlating them with known parameters, such as stellar profile shapes, airmass, $\mathrm{X}$ and $\mathrm{Y}$ pixel position.

Light curves with significant peaks in the BLS spectrum are flagged, and investigated further. We check for out-of-transit variations, such as ellipsoidal variation (Sirko \& Paczyński 2003), the existence of secondary transits, the shape of the light curve, whether the depth and duration are compatible with a planet-sized body (a few $R_{\text {Jup }}$ radius or smaller) orbiting the given star, etc. After an automated filtering procedure is performed, and rather generous cuts are applied, we manually check the candidates on the screen, and promote the best ones for follow-up work. Of the order of 100,000 light curves have been checked in this way (on the screen), and thousands of transits have been found, with only a few hundred considered worthy of further follow-up. We note that the selection criteria and procedures have significantly changed over the past four years.

\section{Follow-up of Candidates}

\subsection{Reconaissance Spectroscopy}

Our primary workhorse is the Digital Speedometer (DS) at SAO's $1.5 \mathrm{~m}$ Tillinghast reflector at FLWO, Arizona (Latham 1992). Initial characterization of the candidate 


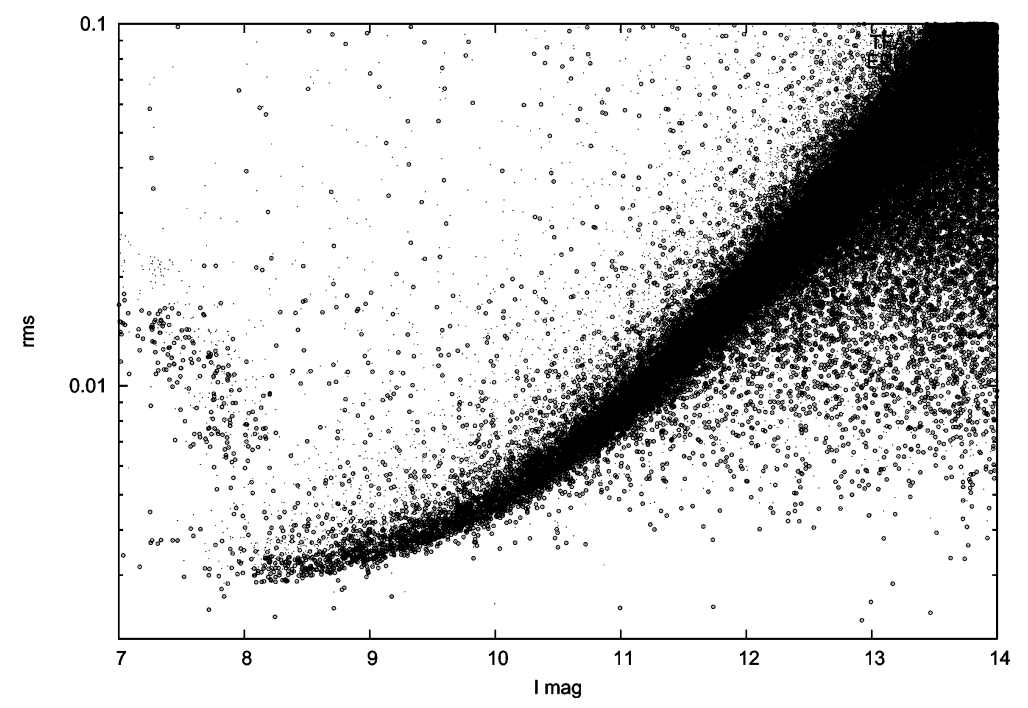

Figure 3. The rms of light curves sampled at 5.5-min cadence as a function of median I-band magnitude. Small black dots represent light curves after "external parameter decorrelation" processing, gray circles show the rms after subsequent TFA (trend-filtering algorithm) processing. Precision reaches 2.9 mmag.

stars provides an estimate of the effective temperature, surface gravity and rotational broadening $(v \sin i)$. Some candidates are excluded after one spectrum, because their low surface gravity $(\log g \lesssim 3.0)$ indicates that they are clearly giants, so that a body orbiting about them on a few day orbit is physically unrealistic. These are either false photometric detections, or blends with an eclipsing binary that falls along the line of sight. Some other targets are also excluded after a single observation if the spectrum is clearly double-lined, and thus due to a grazing eclipsing binary. Most targets, however, need more observations to decipher their nature. Binaries involving an F star orbited by a Jupiter-size M star (F+M binaries) without significant out-of-transit variations are detected by taking two or more DS spectra; their radial velocity typically varies by many $\mathrm{km} \mathrm{s}^{-1}$. Finally, some hierarchical triple stars reveal themselves by moving broad shoulders in the cross-correlation function of the individual spectra with a synthetic template. Triples typically require more follow-up work. We note that massive planets around normal dwarf stars may already be detected by the Digital Speedometer, such as the case of HAT-P-2b (see later Fig. 4) or the early result of Latham et al. (1989).

\subsection{Photometry Follow-up}

Surviving candidates are normally observed with the FLWO $1.2 \mathrm{~m}$ telescope and KeplerCam, or TopHAT. Possible reasons for this step are: i) to decide which star is the true variable in an otherwise crowded field that causes serious blending with HAT's 14 pixel scale, ii) to confirm the photometric variation if that was near the detection threshold, iii) to characterize the light curve, for instance check the shape and depth.

\subsection{High Precision Spectroscopy Follow-up}

The best candidates are promoted for observations with prime instruments, such as HIRES on Keck-I, or the SOPHIE spectrograph at $1.93 \mathrm{~m}$ telescope of the Observatory of Haute-Provence (OHP). For a certain detection, we require a clear radial velocity orbit in phase with the photometric signal, and negligible correlations between phase and line bisector variations. Small bisector variations further strengthen the case. 

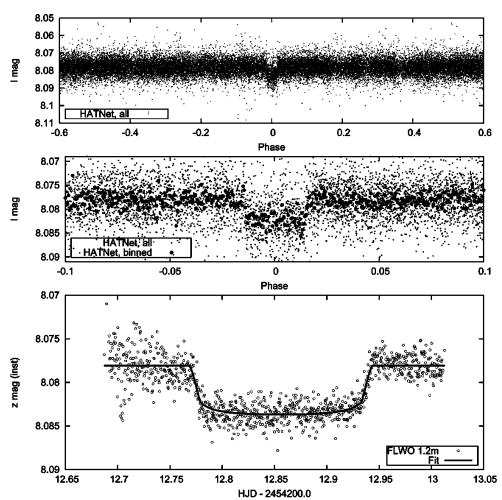

(a)

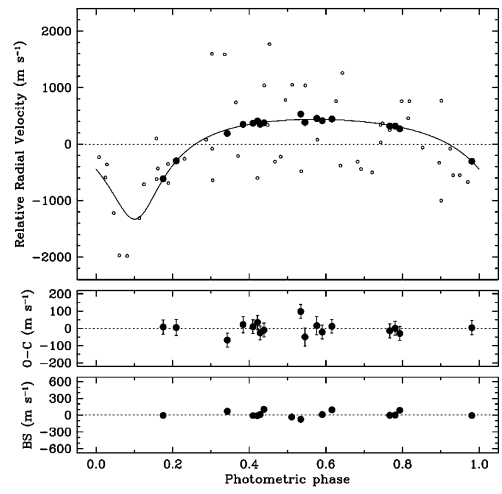

(b)

Figure 4. Discovery data of HAT-P-2b, a super-massive transiting exoplanet detected by HATNet and the Wise-HAT (WHAT) telescopes. (a) The upper panel shows the unbinned light curve with 26400 data-points, phased with the $P=5.63 \mathrm{~d}$ period. The middle panel shows the same data zooming in on the transit and binned. The lower panel displays the Sloan $z$-band photometry taken with KeplerCam on the FLWO $1.2 \mathrm{~m}$ telescope. Overplotted is our best fit obtained with the Mandel \& Agol(2002) formalism. (b) The upper panel shows the RV measurements phased with the same period as used in the right panel. The zero-point in phase corresponds to the epoch of mid-transit. Large filled circles indicate Keck and Lick points. Small open circles denote CfA DS data (not used for the fit). The orbital fit is overlaid assuming $40 \mathrm{~m} \mathrm{~s}^{-1}$ stellar jitter. The middle panel shows the residual of the fit. The lower panel exhibits the line bisector spans on the same scale as the upper panel, showing no significant variation.

\section{Results in a Nutshell}

Impostors that mimic planetary transits will be described in a future paper. We consider these results to be an important outcome of the transit searches that deserves attention.

In 2007, after 3 years of intensively running the HATNet project, the efforts bore fruit, and the first transiting planets appeared in our pipeline. As of mid-2008, planets based on Keck/HIRES observations are HAT-P-1b through HAT-P-4b and HAT-P-6b through HAT-P-8b, whereas planets based on final confirmation by SOPHIE/OHP are HAT-P-5b and HAT-P-9b. We would like to highlight that HATNet, due to its network coverage, is fairly sensitive to long period transiting extrasolar planets. Indeed, at the time of its publication, HAT-P-1b (Bakos et al. 2007a) with a period of $\mathrm{P}=4.46 \mathrm{~d}$ was the transiting planet with the longest known period. The same holds for HAT-P-2b with $\mathrm{P}=5.63 \mathrm{~d}$ period (Bakos et al. 2007b, Fig. 4). Equally important, HATNet has proven to be sensitive to very shallow photometric signals. The smallest amplitude transit that we have detected so far has a depth of $1.8 \mathrm{mmag}$, and the variation was confirmed by the Digital Speedometer as due to a blended eclipsing binary. The transit depth of HAT-P-2b in the combined HATNet and WHAT data reaches a signal-to-noise of 26 .

Some highlights from the HAT planets are presented below.

HAT-P-1b: Orbits one member of a visual binary of G0 main-sequence stars with $10^{\prime \prime}$ separation, corresponding to $1550 \mathrm{AU}$ at the estimated distance of $140 \mathrm{pc}$. The two stellar components are merged on the HATNet frames, and cause an elongated blend with poor photometry. Nevertheless, the TFA method improved the light curve so much that it led to a clear detection, and subsequent confirmation with Keck/HIRES (Bakos et al. 2007a). HAT-P-1b is a low-mass $\left(0.53 M_{\mathrm{J}}\right)$ hot Jupiter with somewhat inflated radius 


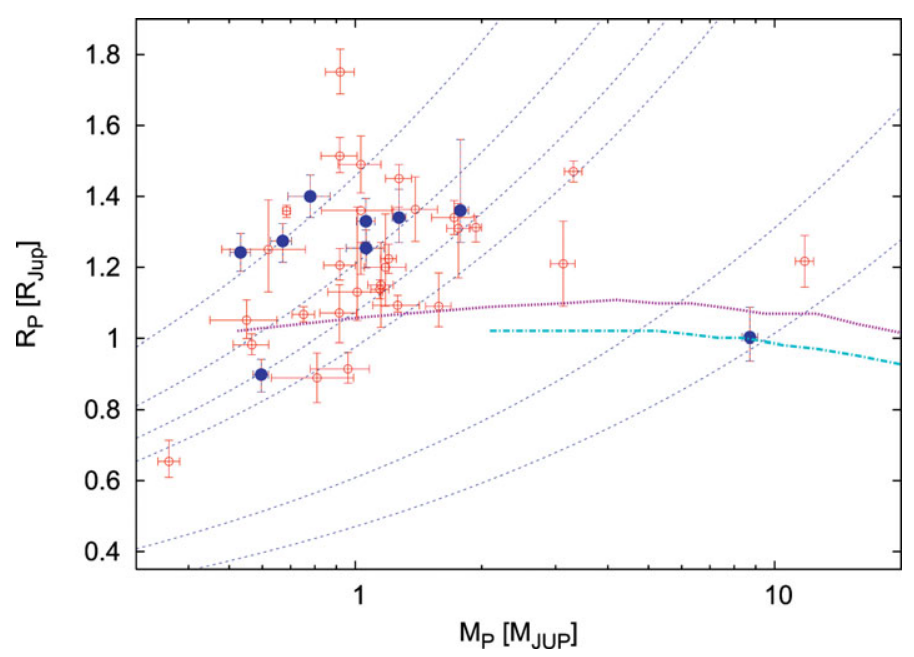

Figure 5. Mass versus radius diagram for published transiting extrasolar planets as of June 2008. Filled circles represent HATNet's contribution.

$\left(1.2 R_{\mathrm{J}}\right)$. At the time of discovery, by joining HD $209458 \mathrm{~b}$ as another inflated planet, it highlighted that the latter is not an exception, but "bloated" planets may be frequent.

HAT-P-2b: A massive and eccentric $(e \approx 0.5)$ planet around a bright star $(\mathrm{V}=8.7)$. HAT-P-2b (Bakos et al. 2007b) is an "outlier" among the transiting planets in Fig. 5, having a mass of about $8 M_{\mathrm{J}}$, even though its radius is close to $1 R_{\mathrm{J}}$, implying that its mean density is that of lead; furthermore it orbits its parent star at $P \approx 5.6 d$ with such high eccentricity that its insolation varies by a factor of 9 over its orbit.

HAT-P-3b: A fairly compact transiting hot Jupiter that orbits a metal-rich early K dwarf with period of 2.90 days (Torres et al. 2007). The relatively small size $(0.89 \pm$ $\left.0.05 R_{\mathrm{J}}\right)$ of the object for its mass $\left(0.60 \pm 0.03 M_{\mathrm{J}}\right)$ implies the presence of about $75 M_{\oplus}$ worth of heavy elements ( $1 / 3$ of the total mass) based on current theories of irradiated extrasolar giant planets.

HAT-P-4b: A low-density extrasolar planet transiting a $V \approx 11.2$, slightly evolved metal-rich late $\mathrm{F}$ star with period of 3.05 days. The planet has a mass of $0.68 \pm 0.04 M_{\mathrm{J}}$, and radius of $1.27 \pm 0.05 R_{\mathrm{J}}$, clearly belonging to the group of "inflated" extrasolar planets (Kovács et al. 2007).

HAT-P-5b: A roughly Jupiter mass and $1.26 \pm 0.05 R_{\mathrm{J}}$ size planet with period of 2.79 days, confirmed by the SOPHIE spectrograph at OHP (Bakos et al. 2007c).

HAT-P-6b: A giant planet, with radius $1.33 \pm 0.06 R_{\mathrm{J}}$ and mass $1.06 \pm 0.12 M_{\mathrm{J}}$, transiting a bright $(\mathrm{V}=10.5)$ late $\mathrm{F}$ star with $\mathrm{P}=3.85 \mathrm{~d}$ period (Noyes et al. 2008).

HAT-P-7b: A very hot giant planet orbiting a bright ( $\mathrm{V}=10.5)$ slightly evolved F6 star in Kepler's field with a small semi-major axis of $a=0.0377 \mathrm{AU}$. It is a fairly massive $\left(1.78 \pm 0.05 M_{\mathrm{J}}\right)$ planet with radius of $1.36 \pm_{0.09}^{0.20} R_{\mathrm{J}}$. The relatively hot and large host star, combined with the close orbit of the planet, yield a very high planetary irradiance. If the planet re-radiates its absorbed energy before distributing it to the night side, the day-side temperature should be about $2700 \mathrm{~K}$ (Pál et al. 2008).

HAT-P-8b: As compared to the average of known transiting exoplanets, HAT-P-8b is a relatively large radius $\left(1.3 R_{\mathrm{J}}\right)$ and massive $\left(1.26 M_{\mathrm{J}}\right)$ planet with $3.07 \mathrm{~d}$ period (very similar to HAT-P-4b), orbiting a $1.2 M_{\odot}$ star with $6200 \mathrm{~K}$ effective temperature (Latham 2008, submitted). 
HAT-P-9b: This is a planet transiting a moderately faint $(\mathrm{V}=12.3 \mathrm{mag})$ late $\mathrm{F}$ star, with an orbital period of 3.92 days. The radius $\left(1.40 \pm 0.06 R_{\mathrm{J}}\right)$ and mass $\left(0.78 \pm 0.09 M_{\mathrm{J}}\right)$ lead to a very low density of $\rho=0.35 \pm 0.06 \mathrm{~g} \mathrm{~cm}^{-3}$ (Shporer et al. 2008).

\section{Acknowledgements}

We are indebted to Bohdan Paczyński for initiating the HAT project, and supporting it over the years, to Grzegorz Pojmański for openly sharing his original design with our group, and to Kris Stanek for his encouragement during early phases of the project. We would like to thank Carl Akerlof and the de-commissioned ROTSE-I project the generous loan of some of the lenses and CCDs that we use for operating HATNet. We owe special thanks to Emilio Falco, Dan Fabricant, James Moran, Antony Schinckel and Ray Blundell for their help in establishing and operating the HATNet stations at FLWO and SMA. We are grateful for our collaborators D. Fischer, G. Marcy, P. Butler for their contribution to Keck/HIRES observations and analysis, and to F. Bouchy, F. Pont, T. Mazeh and A. Shporer for their collaboration using the SOPHIE spectrograph. We wish to thank the support given by telescope operators Mike Calkins and Perry Berlind in the operation of the FLWO HATNet station.

\section{References}

Bakos G. Á. et al. 2002, PASP, 114, 974

Bakos G. Á. et al. 2004, A\&AP, 116, 266

Bakos, G. Á., et al. 2007, ApJ, 656, 552

Bakos, G. Á., et al. 2007, ApJ, 670, 826

Bakos, G. Á., et al. 2007, ApJ, 671, L173

Gaudi, S. 2006, ASP Conf. Ser., astroph/12141

Kovács, G., Zucker, S., \& Mazeh, T. 2002, A\&AP, 391, 369

Kovács, G., Bakos, G. Á., \& Noyes, R. W. 2005, MNRAS, 356, 557

Kovács, G., et al. 2007, ApJ, 670, L41

Latham, D. W., Stefanik, R. P., Mazeh, T., Mayor, M., \& Burki, G. 1989, Nature, 339, 38

Latham, D. W. 1992, ASP Conf. Ser., 32, 110,

Mandel, K. \& Agol, E. 2002, ApJ, 580, L171

Noyes, R. W., et al. 2008, ApJ, 673, L79

Robin et al. 2003, A\&AP, 409, 523

Pál, A. \& Bakos, G. Á., 2006, PASP, 118, 1474

Pál, A., et al. 2008, ApJ, 680, 1450

Pojmański, G. 1997, Acta Astronomica, 47, 467

Pont, F., Zucker, S., \& Queloz, D. 2006, MNRAS, 373, 231

Shporer, A., et al. 2008, astroph/0806.4008

Sirko, E. \& Paczyński, B. 2003, ApJ, 592, 1217

Skrutskie, M. F. et al., 2006, AJ, 131, 1163

Torres, G., et al. 2007, ApJ, 666, L121 